\title{
Oral drug therapy in elderly with dysphagia: between a rock and a hard place!
}

This article was published in the following Dove Press journal:

Clinical Interventions in Aging

31 January 2017

Number of times this article has been viewed

\author{
Serena Logrippo',2 \\ Giovanna Ricci ${ }^{3}$ \\ Matteo Sestili ${ }^{4}$ \\ Marco Cespi ${ }^{2}$ \\ Letizia Ferrara ${ }^{4}$ \\ Giovanni F Palmieri² \\ Roberta Ganzetti ${ }^{4}$ \\ Giulia Bonacucina ${ }^{2}$ \\ Paolo Blasi \\ 'School of Advanced Studies, \\ ${ }^{2}$ School of Pharmacy, ${ }^{3}$ School of Law, \\ University of Camerino, Camerino, \\ ${ }^{4}$ Italian National Research Centers on \\ Ageing (INRCA), Ancona, Italy
}

Correspondence: Giulia Bonacucina School of Pharmacy, University of Camerino, Via Gentile III Da Varano, Camerino 62032, Italy

Tel +390737402289

Fax +390737402727

Email giulia.bonacucina@unicam.it

Roberta Ganzetti

Italian National Research Centers on Ageing (INRCA), Via della Montagnola, 8I, Ancona 60I27, Italy

Tel +390718003150

Fax +39 07I 8003313

Email r.ganzetti@inrca.it

\begin{abstract}
Demographic indicators forecast that by 2050, the elderly will account for about one-third of the global population. Geriatric patients require a large number of medicines, and in most cases, these products are administered as solid oral solid dosage forms, as they are by far the most common formulations on the market. However, this population tends to suffer difficulties with swallowing. Caregivers in hospital geriatric units routinely compound in solid oral dosage forms for dysphagic patients by crushing the tablets or opening the capsules to facilitate administration. The manipulation of a tablet or a capsule, if not clearly indicated in the product labeling, is an off-label use of the medicine, and must be supported by documented scientific evidence and requires the patient's informed consent. Compounding of marketed products has been recognized as being responsible for an increased number of adverse events and medical errors. Since extemporaneous compounding is the rule and not the exception in geriatrics departments, the seriousness and scope of issues caused by this daily practice are probably underestimated. In this article, the potential problems associated with the manipulation of authorized solid oral dosage forms are discussed.
\end{abstract}

Keywords: geriatric medicine, dysphagia, compounding, modified-release formulations, gastrointestinal tract toxicity

\section{Introduction}

Biodemography scientists have registered a significant lengthening of lifespan in the last 50 years. This is essentially due to the progress made in medicine and public health. Counterintuitively, the morbidity among elderly is continuously growing and the need for medical care increases with geriatric patient age. ${ }^{1}$ Also, considering the limits due to unpredictable happenings and the absence of reliable old records, the demographic trend draws a scary picture. It is estimated that, by 2050 , people aged 65 or more will account for about $25 \%$ of the total population of developed countries and $15 \%$ of the people living in developing countries. ${ }^{2}$ This change in society composition will have strong repercussions in many societal policies, and a huge impact is expected in medical and health care system.

Medicine faces the great responsibility to deal with a continuously growing elderly population, and geriatric medicine is (and will be) the main actor of this revolution. A geriatric patient has many peculiar features that challenge the everyday work of clinicians, pharmacists, nurses, and health care providers (HCPs). Due to the aging process, the elderly show different pharmacokinetics and pharmacodynamics with respect to young adults, ${ }^{3}$ and the presence of different pathologic conditions (also known as comorbidity) obliges geriatric physicians to prescribe a large number of medicines, putting the patient in polypharmacotherapy. This situation further complicates the scenario. ${ }^{4,5}$ 
Difficulty in swallowing and dysphagia may be seen as a marginal problem, considering the acute and chronic diseases that these patients have to deal with. ${ }^{6}$ Unfortunately, this is not the case. Dysphagia is most common among the elderly population because the aging process can negatively affect the oral, pharyngeal, and esophageal phases of the swallowing process. It can become worse by reduced saliva production. ${ }^{7,8}$ Difficulty in swallowing may be responsible for malnutrition, weight loss, and dehydration. At the same time, food and/or liquid entering the airways can provoke respiratory infections. ${ }^{9}$

In patients over 65 years of age, the prevalence of dysphagia ranges from $7 \%$ to $13 \%,{ }^{10-12}$ a percentage that increases with age and if patients are affected by stroke, postoperative cognitive dysfunction, or neurodegenerative diseases such as Parkinson's and dementia. ${ }^{913-17}$ Worthy of note is the fact that swallowing difficulties have been reported in about $50 \%$ of patients in nursing homes. ${ }^{18}$

Since the elderly patients are intrinsically predisposed to dysphagia and the number of elderly is increasing, the number of dysphagic patients will increase in the near future. This global demographic trend, fueled by falling birth rates and the continuous increase in life expectancy, is now certain, and brings with it significant social distress and a variety of chronic disabilities that will challenge health care systems and increase the need for pharmacologic and surgical treatments. ${ }^{19,20}$

Elderly patients with dysphagia are between a rock and a hard place, because they require a large number of prescriptions like other geriatric patients, ${ }^{4,5,21}$ but difficulties with swallowing or dysphagia limit or preclude the administration of solid oral dosage forms, which are by far the most common formulations on the market. ${ }^{22}$ The problem could easily be bypassed if all the active pharmaceutical ingredients (APIs) contained in marketed products were available in formulations other than solid oral dosage forms. Unfortunately, this is not the case, and in clinics, compounding is a daily practice as caregivers dispense crushed tablets or opened capsules to facilitate the administration of solid oral dosage forms to dysphagic patients. ${ }^{23,24}$

The US Food and Drug Administration defines compounding as

A practice in which a licensed pharmacist, a licensed physician, or, in the case of an outsourcing facility, a person under the supervision of a licensed pharmacist, combines, mixes, or alters ingredients of a drug to create a medication tailored to the needs of an individual patient. ${ }^{25}$
Tablet and capsule manipulation can include tablet splitting, tablet crushing, and capsule opening, followed by the dispersion/dissolution of the tablet material or capsule content (eg, powder or granules) in water, beverages, gels, or food to allow better deglutition. ${ }^{26}$ When deglutition is completely impaired, the solution, suspension, or low-viscosity gel is delivered directly into the stomach by an enteral tube. The manipulation of a tablet or a capsule can seem trivial and insignificant. ${ }^{27}$ On the contrary, this is absolutely untrue: compounding of marketed products has been recognized as responsible for adverse events and medical errors. ${ }^{28-32}$

Representative is the title of a paper recently published by Verrue et $\mathrm{al}^{33}$ on tablet splitting "Tablet-splitting: a common yet not so innocent practice". This sentence resumes quite well what happens in the everyday nursing practice. In fact, tablet splitting is, most of the time, an off-label use of the medicinal product and, even if it can be considered a priori safer than tablet crushing, it can have a negative impact on therapy outcome.

Before reaching the market, a medicinal product is carefully evaluated for its safety and efficacy through clinical trials, and all the technical data are reviewed and evaluated by the appropriate regulatory agency. Obviously, safety and efficacy are guaranteed if the product is used on-label, that is, by following the information reported in its labeling that includes product information leaflet and summary of the product characteristics. From the legislative point of view, the manipulation of a marketed formulation is defined as an off-label use of the drug. In fact, when a medicinal product is approved by the regulatory authorities, it can be administered to treat the diseases listed in the product labeling at the specified dose regimens and route of administration. This applies to the dosage form as well. Any manipulation of an approved medicinal product, such as by tablet splitting or crushing, or change in the administration route from oral to intragastric is, if not specified in the product information leaflet and summary of the product characteristics, an off-label use of the medicine. In these circumstances, the prescriber, the pharmacist, and the nurse may become legally responsible for any adverse effect resulting from taking the medicine. ${ }^{34}$

Compounding can affect the biopharmaceutical features of the product and its therapeutic outcome, resulting in an increased probability of adverse reactions for the patient. ${ }^{35}$ Patient risk is particularly important for APIs with a narrow therapeutic window, for drugs that are irritant to the gastrointestinal tract (GIT) mucosa, for drugs formulated in modified-release solid oral dosage forms, and for any product that is hormonal or steroid-based, cytotoxic, and teratogenic. ${ }^{36,37}$ For this reason, 
the act of manipulating a solid dosage form should be undertaken only if is impossible to give the medicine by an alternative route, or in a different licensed formulation. ${ }^{38}$

Given the threats associated with the practice of compounding marketed medicinal products and its wide use for dysphagic patients in hospital geriatric units and home care, we believe that the risks for the patients and the ethical and legal issues connected to these procedures are underestimated and worthy of discussion.

This review focuses on concerns related to the manipulation of medicinal products and concentrates its attention on biopharmaceutical/pharmacokinetics issues and GIT toxicity that may derive from this practice.

\section{Biopharmaceutical considerations}

Biopharmaceutics has a fundamental role in rationalizing and improving medicinal product performances and should be taken into consideration when compounding is contemplated.

Once a solid dosage form is administered orally to patients, the API has to be released and/or dissolved from the dosage form to the body fluids in order to be absorbed and distributed in the various organs and tissues. Virtually, no API possesses adequate characteristics to be administered on its own to patients, and its formulation in an adequate dosage form is needed. During the formulation step, an API is combined with different excipients and, with the aid of ad hoc technologic procedures, is shaped into the final medicinal product that has the required features for administration through the chosen route. The API can be formulated in a variety of dosage forms to obtain medicinal products with the desired consistency: solid (tablets, capsules, granules), semisolid (creams, ointments), or liquid. Solid dosage forms are the leading formulations on the market, and of these, $>60 \%$ of APIs intended for oral administration are formulated as tablets and capsules.,22

The oral route is highly preferable because patients can take the medicine themselves and, accordingly, it is associated with superior patient compliance and adherence. Solid dosage forms are also preferred by pharmaceutical companies because not only does the API stability tend to be higher in the solid state but also they cost much less to manufacture than the parenteral forms. Thus, solid dosage forms are the first choice for oral administration. ${ }^{22}$

The predominance of solid oral dosage forms, especially tablets and capsules, is the leading cause of potential issues in dysphagic patients. In fact, when administration through a different route is not possible and/or a different formulation is not available, the physician and the pharmacist face an important decision: should they prepare an ad hoc formulation in the hospital pharmacy or in an accredited laboratory, or should they manipulate the commercial formulation? Obviously, the best choice is to set up an ad hoc formulation, but this is not always possible since some APIs, especially those most recently approved, are not available in bulk. At the moment, the compounding of marketed medicinal products is the most common method for administering medication to dysphagic patients in hospitals and clinics, but the safety risks it poses have been largely underestimated. 23,39,40

Crushing a solid dosage form in a mortar cannot be considered satisfactory from the point of view of reproducibility and quality control. API particle size should be measured to guarantee at least the reproducibility of the manipulation procedure, but the presence of excipients makes the measurement challenging. ${ }^{41}$ API particle size has a direct relation to the dissolution rate and apparent solubility, and this correlation is particularly significant for low water-soluble compounds. ${ }^{42}$ Because of this, different particle sizes or particle size distributions generate diverse plasmatic curves and pharmacologic effects. ${ }^{43}$

Today, the presence of many modified-release formulations in the market makes the decision "whether to crush a tablet or not" more difficult and much more dangerous. So, compounding should only be recommended by HCPs with sufficient knowledge of biopharmaceutics and the proprietary information about the technology used to prepare the tablet or capsule. In fact, the first step for assessing the actual risks for the patient is to distinguish between a conventional-release (also called immediate-release) and a modified-release dosage form.

Conventional-release dosage form compounding may generate faster absorption and higher bioavailability (Figure 1). ${ }^{44,45}$ These issues become more significant and extremely dangerous for the geriatric dysphagic patient in the case of drugs with a narrow therapeutic index, such as warfarin, carbamazepine, digoxin, lithium, and theophylline, for which small changes in systemic concentration can lead to significant changes in pharmacodynamics. ${ }^{46}$ Additional problems may come from the imprecision of the dose, the reduced stability in solution, and the mixing with food, beverages, or viscous gels to facilitate swallowing.

While the manipulation of conventional-release medications presents dangers, even greater risk is posed by compounding of modified-release dosage forms, which considerably affects the product biopharmaceutics with harmful consequences for the patient. In general, the compounding of modified-release dosage forms is not recommended and 


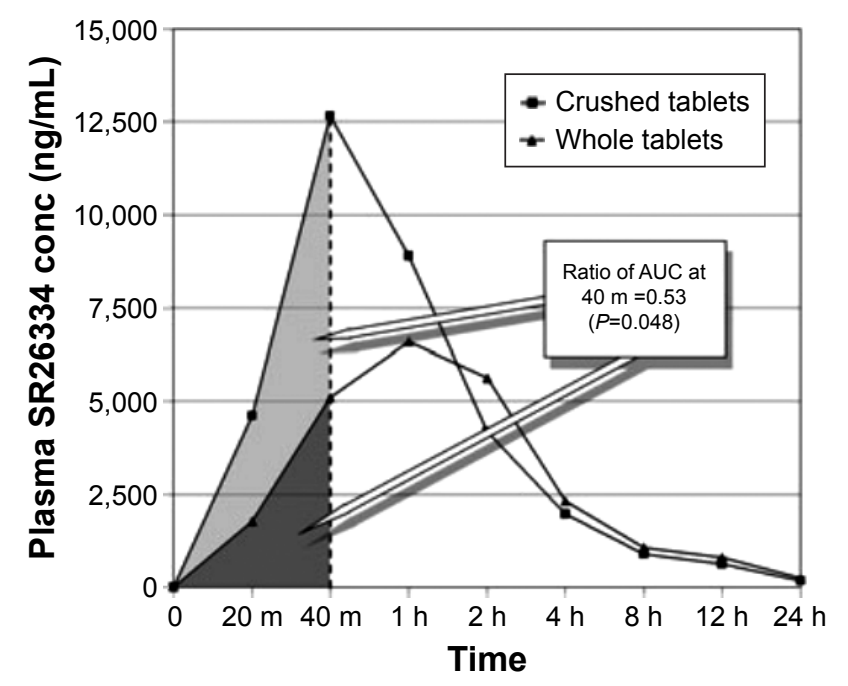

Figure I Median plasma concentrations of clopidogrel metabolite SR26334 following administration of whole or crushed $300 \mathrm{mg}$ clopidogrel tablet.

Notes: The crushed tablet has been administered through a nose-gastric tube. Reproduced from Zafar MU, Farkouh ME, Fuster V, Chesebro JH. Crushed clopidogrel administered via nasogastric tube has faster and greater absorption than oral whole tablets. J Interv Cardiol. 2009;22(4):385-389, with permission from John Wiley and Sons. Copyright 2009.44

Abbreviations: AUC, area under the curve; conc, concentration.

should be highly discouraged. ${ }^{47,48}$ In these dosage forms, compounding can strongly alter not only the amount of API released over time but also its release site, affecting its efficacy and toxicity potential. Useful resources in this regard are "Oral Dosage Forms That Should Not Be Crushed 2015"48 and the "Don't Rush to Crush" handbook. ${ }^{49}$

In the case of prolonged-release oral solid medications, which are designed to release the API over an extended period of time, administration of a crushed form results in rapid absorption of the entire dose with adverse outcomes for the patient. ${ }^{36,50}$ Enteric-coated formulations of acid-labile APIs are designed to pass through the stomach intact and deliver the drug in the intestines. Crushing the medications subverts the intention of the formulators because the drug is then inactivated by gastric acid. ${ }^{51,52}$

With the availability of many modified-release solid oral dosage forms on the market, the "to crush or not to crush" dilemma is extremely critical. In fact, albeit a recent European Medical Agency guideline states,

If applicable, specific recommendations should be provided

to ensure optimum conditions of use (eg, instructions not

to chew or crush tablets, etc),

most of the marketed modified-release formulations do not have the "not to chew or crush" recommendation. ${ }^{53}$

Issues connected to the compounding of a solid oral dosage form are manifold, but "dose dumping" is certainly the most serious consequence, since it presents the highest risk of morbidity and mortality for the patient. Dose dumping can be defined as

an unintended, rapid drug release of the entire amount or a significant fraction of the active substance contained in a modified release dosage form. ${ }^{54}$

As an example, we can briefly mention the story of an 83-year-old female who died after 3 weeks of chewing rather than swallowing the intact prolonged-release diltiazem capsule, because she found it difficult to swallow. ${ }^{55}$ In another case study, Schier et al ${ }^{50}$ reported the death of a woman after repeated daily administration of crushed prolonged-release nifedipine tablet through a nasogastric tube. Her death was ascribed to severe hypotension caused by high plasma concentration of nifedipine generated by the immediate release of the entire $90 \mathrm{mg}$ dose (ie, dose dumping), which instead was formulated for release over an extended period of time.

However, some modified-release dosage forms have been designed to allow compounding. In a recent study, healthy subjects were administered intact or crushed capsules containing microencapsulated oxycodone or immediate-release oxycodone tablets. ${ }^{56}$ Once the capsule is opened and the microparticles are crushed, the medicine remains bioequivalent with the capsule swallowed intact (Figure 2): this makes the formulation suitable for compounding for dysphagic patients. On the contrary, crushed modified-release tablets become bioequivalent to the powder of the immediate-release oxycodone (Figure 2).

As is evident, not all solid dosage forms are suitable for splitting or crushing. But even when compounding is permissible, human error by HCPs may dissipate the product and alter the dose administered, which can have serious clinical consequences, especially for APIs with a narrow therapeutic index. ${ }^{33,57}$ Even though the problem of oral drug therapy in geriatric dysphagic patients is not new, most scientists in academia and industry do not seem very sensitive to this issue. In a recent literature search, Messina et a $1^{58}$ evidenced the complete absence of scientific publications addressing the age appropriateness of medicinal products for the elderly. Regulatory agencies in North America and Europe are slowly moving the first step toward the elaboration of ad hoc strategies for the development of geriatric medicines. Unfortunately, no guidelines on the adequacy of dosage forms to geriatric patients are available at the moment. ${ }^{59}$

\section{Damage to patient GIT}

Drug toxicity in the GIT is a common and important medical problem linked to extemporaneous compounding that can affect part or all of the GIT, with consequences ranging from 

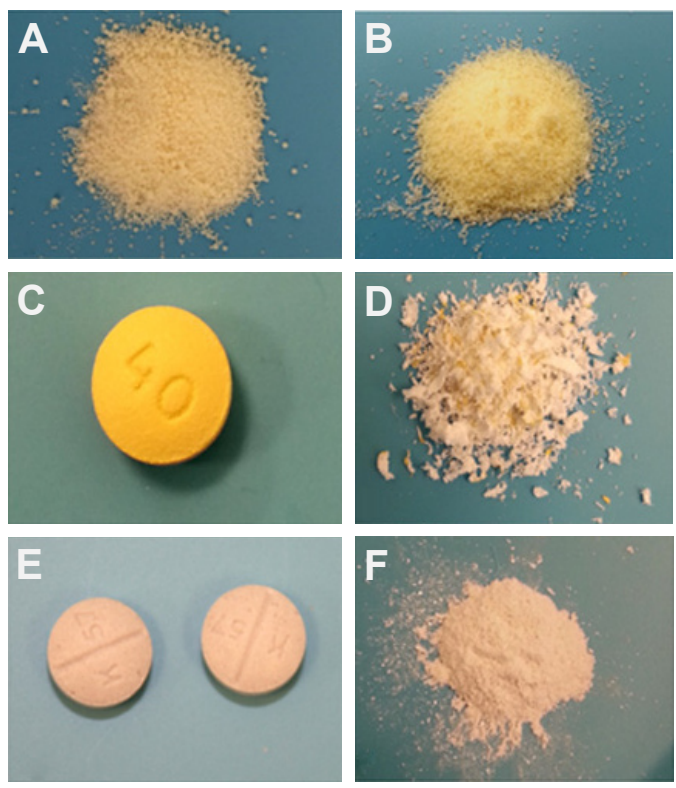

\section{DETERx}

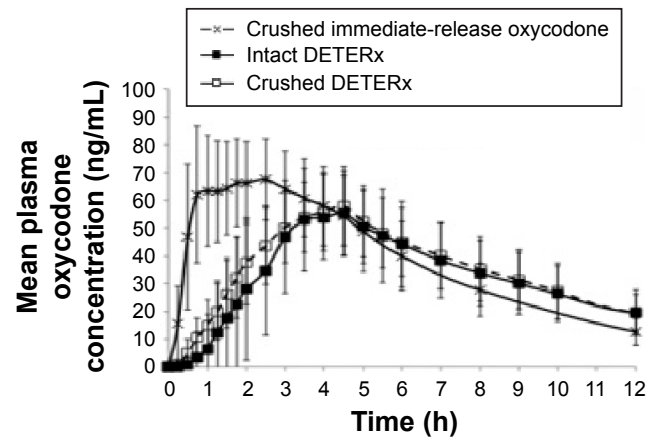

OxyContin

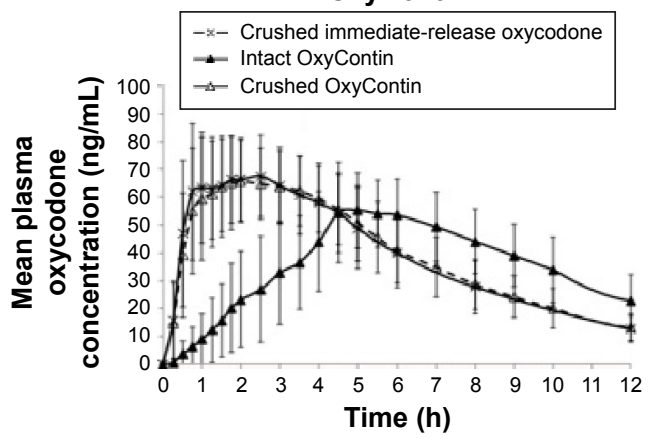

Figure 2 Left side: (A) intact microspheres ( $40 \mathrm{mg}$ ) obtained by empting one capsule of Deterx ${ }^{\circledR}$; (B) crushed microspheres (40 mg) from one capsule of Deterx; (C) OxyContin ${ }^{\circledR}$ tablet intact $(40 \mathrm{mg})$; (D) Oxycontin tablet crushed $(40 \mathrm{mg})$; (E) two $20 \mathrm{mg}$ intact immediate-release oxycodone tablets; (F) powder from two crushed immediate-release oxycodone tablets. Right side: mean plasma concentration-time curve profiles generated by the administration of intact and crushed Deterx compared with crushed immediate-release oxycodone (upper figure); mean plasma concentration-time curve profiles generated by the administration of intact and crushed Oxycontin compared with crushed immediate-release oxycodone (lower figure). Reproduced from Gudin J, Levy-Cooperman N, Kopecky EA, Fleming AB. Comparing the effect of tampering on the oral pharmacokinetic profiles of two extended-release oxycodone formulations with abuse-deterrent properties. Pain Med. 20I5; I6(II):2I42-2I5I, by permission of Oxford University Press. ${ }^{56}$

minor pathologies such as drug-induced diarrhea to perforation or fatal hemorrhage. ${ }^{60}$ The morbidity and associated medical costs due to drug-induced GIT toxicity, as well as the mortality that may ensue, are probably underestimated.

A number of APIs, including ferrous sulfate, ${ }^{61}$ bisphosphonates, ${ }^{62}$ potassium chloride, ${ }^{63}$ nonsteroidal antiinflammatory agents (NSAIDs), ${ }^{64}$ and tetracycline, ${ }^{65}$ have been identified as being responsible for GIT mucosal injuries. Sample endoscopic and microscopic views of GIT mucosae damaged by NSAIDs are shown in Figure 3.

To provide protection from these APIs, formulators use gastro-resistant enteric coatings made of polymers that remain intact in the stomach but dissolve and release the API in the more alkaline $\mathrm{pH}$ of the small intestine. Enteric
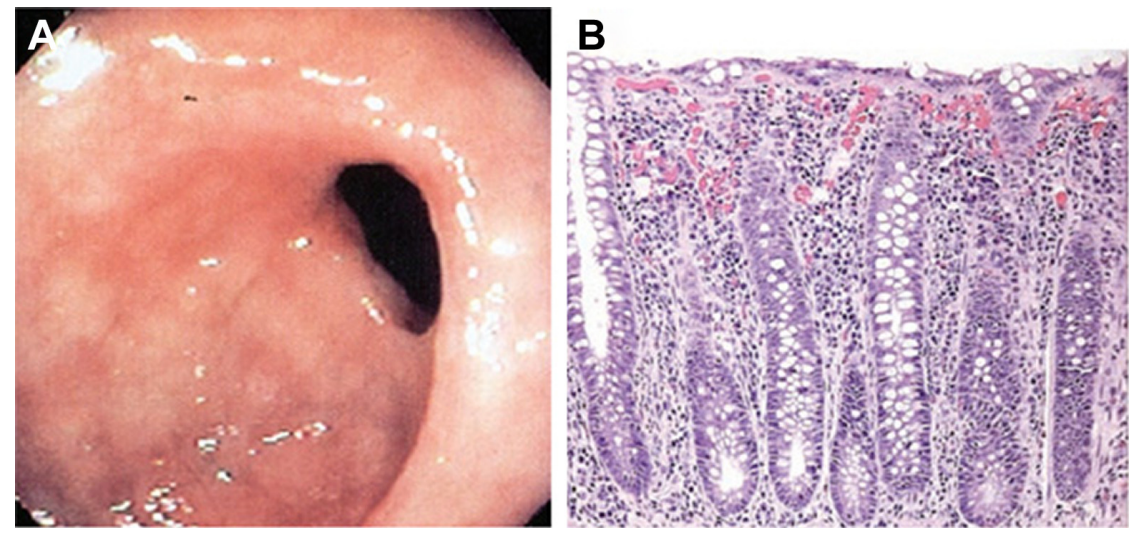

Figure 3 Sample endoscopic and microscopic views of gastrointestinal tract mucosae damaged by NSAIDs.

Notes: (A) Endoscopic view of a stricture in the cecum of the colon in a patient after long-term NSAID use. The stricture has resulted in significant narrowing of colonic lumen. Such diaphragms have also been reported to occur in the small intestine. (B) Histologic section from a colonic biopsy showing a moderate inflammatory infiltrate in the lamina propria, mild lymphocytic infiltration of the glandular epithelium, and scattered eosinophils. Adapted by permission from Macmillan Publisher Ltd: Nat Clin Pract Gastroenterol Hepatol, Pusztaszeri MP, Genta RM, Cryer BL, Drug-induced injury in the gastrointestinal tract: clinical and pathologic considerations, 2007;4(8):442-453, copyright 2007. ${ }^{74}$

Abbreviation: NSAID, nonsteroidal anti-inflammatory drug. 
coatings not only serve to prevent stomach irritation but also make it possible to delay the release of APIs that are inactivated by the stomach contents, or to delay the onset of action at a specific site within the GIT.

Gastrointestinal symptoms resulting from either prescribed medications or over-the-counter drugs are frequently encountered in geriatric practice. The manipulation of solid oral dosage forms to allow easy administration in dysphagic patients may increase the incidence and severity of adverse reactions involving the upper and lower GIT.

We subsequently discuss the APIs used in elderly patients that are responsible for GIT mucosal injuries, especially when the formulations are manipulated to allow swallowing.

\section{Ferrous sulfate}

Liabeuf et $\mathrm{al}^{61}$ reported a series of cases of ulceration of the oral mucosa linked to direct contact with ferrous sulfate in elderly patients, particularly when swallowing disorders were present, and advised that appropriate pharmaceutical formulations (eg, syrups) should be administered to at-risk patients. The mucosal damage caused by high local iron concentrations may be related to the formation of reactive oxygen species. ${ }^{66}$ Indeed, these species and free radicals have been implicated in mucosal alterations in gastric or intestinal injuries. The mucosal toxicity of ferrous sulfate has also been reported for the hypopharynx, ${ }^{67}$ the esophageal lumen, ${ }^{68}$ and the tracheobronchial tree. ${ }^{69}$ Mouth ulcerations and necrosis have been found after administering crushed tablets containing ferrous sulfate. ${ }^{61}$

\section{Bisphosphonates}

Bisphosphonates are a class of drugs developed over the past 20 years, primarily as antiresorptive agents for treating diseases related to bone remodeling. Soon after the release of alendronate, esophagitis and esophageal strictures were reported, and consequently, the drug labeling had to be changed. Subsequent endoscopic studies in healthy subjects showed that alendronate also caused gastric erosions and ulcers. Persistent mouth ulcerations have been reported by patients who sucked bisphosphonate tablets or let them dissolve in their mouth. ${ }^{70}$ Alendronate has the potential to cause ulcers within the esophagus and stomach, as well as occasional esophageal strictures. Although controlled trials have failed to demonstrate an increased incidence of these adverse events, several case studies and reports have described exudative and ulcerative esophagitis in patients who strictly adhered to recommended use directions, which resolved upon cessation of the therapy. ${ }^{62}$
Risedronate, a third-generation bisphosphonate, seems to be considerably less ulcerogenic and fibrogenic than alendronate and may be a safer alternative for those unable to tolerate alendronate. ${ }^{62}$ In summary, given all the side effects described earlier for this class of drugs, it is recommended that tablets be swallowed whole, and are never split, chewed, or crushed, even in the case of dysphagic patients.

\section{Potassium chloride}

Drug-induced esophagitis caused by potassium chloride is usually present at the junction of the middle and distal thirds of the esophagus above the level of an enlarged heart compressing the distal esophagus. This compound can also lead to the development of strictures, most frequently at or above the level of an enlarged left atrium. ${ }^{63}$

Potassium chloride causes small bowel ulceration as a result of local vascular injury and thrombosis. ${ }^{71}$ Zografos et $\mathrm{al}^{72}$ analyzed 650 cases of drug-induced esophagitis from 1970 to 2009 and reported that five deaths were related to potassium-induced esophageal injury. In particular, these patients developed fistulas from the esophagus into the aorta or left atrium, perforation into the mediastinum, bleeding ulcer, or inanition related to the esophageal stricture. The appearance of dysphagia or swallowing difficulties should be taken seriously for review of oral potassium therapy. It is recommended that oral potassium chloride supplements in liquid form should be administered to patients with cardiomegaly and disorders of esophageal motility. ${ }^{71}$

Quite interestingly, Sinar et $\mathrm{al}^{73}$ performed a controlled multicenter investigation of the effects of oral potassium chloride supplements on the gastrointestinal mucosa. One hundred and twenty healthy men with no endoscopically apparent gastrointestinal lesions were confined to a research ward for 18 days. After the treatment was completed, endoscopic examinations of the esophagus, stomach, and duodenum were performed and evaluated by specialists blinded to the particular treatment given. Mild to moderate gastrointestinal irritation, characterized by erythema and edema, was found with similar frequency in all four treated groups.

\section{Nonsteroidal anti-inflammatory drugs}

Much attention has been focused on NSAIDs, the most commonly prescribed therapeutic class associated with GIT toxicity. In fact, NSAIDs can damage every level of the GIT from the esophagus to the large intestine. ${ }^{64,74}$

Among geriatric patients, the risk of NSAID-induced gastrointestinal complications due to long-term use may be as high as $15 \% .^{75}$ Although NSAIDs cause damage throughout 
the GIT, the predominant gastrointestinal site of injury is the stomach. NSAIDs produce two types of mucosal injury in the stomach. ${ }^{76}$ The first type of damage occurs shortly after the ingestion of the agent and seems to result primarily from local effects on the gastric mucosa (hyperemia, erosions, and/ or subepithelial hemorrhages). The second type of lesion induced by NSAIDs is gastric ulceration, and it is due to their systemic effect on prostaglandin synthesis through inhibition of cyclooxygenase. The clinical consequences of utmost concern among NSAID users are symptomatic gastrointestinal ulcers and ulcer complications such as severe gastrointestinal bleeding and perforation.

In contrast to what happens in the stomach, direct contact of NSAIDs with the intestinal mucosa has a major role in the pathogenesis of NSAID toxicity in the small intestine. Aspirin and nabumetone are rapidly absorbed in the stomach and duodenum and do not undergo enterohepatic recirculation. By contrast, compounds such as indomethacin that are metabolized through enterohepatic cycling are associated with greater intestinal toxicity, even when administered parenterally. ${ }^{77}$

NSAIDs can cause diffuse intestinal inflammation and increased intestinal mucosal permeability, a pathologic condition known as NSAID enteropathy, clinically characterized by occult blood loss, iron deficiency, anemia, malabsorption, and protein loss. ${ }^{78}$

NSAID-induced lesions can develop in the healthy colon or at sites of preexisting colonic disease, such as diverticular disease or chronic inflammatory bowel disease. In some cases, NSAID therapy can reveal previously undiagnosed bowel disease. The most common pattern of NSAID-associated colonic damage is a nonspecific colitis. ${ }^{79}$

To prevent recurrent damage to GIT mucosae, particularly at the esophageal level, a joint effort of patients, physicians, nurses, and pharmacists is required. All patients should be advised to take the medicine only when sitting or standing, take water, and remain upright for at least an hour after administration. ${ }^{80}$ These warnings are of particular importance in elderly patients with dysphagia, or impaired mobility, such as bedridden patients. Several formulations based on NSAIDs are enteric-coated to reduce stomach irritation and minimize drug degradation. Also, in this case, crushing an enteric-coated medication exposes the GIT to the risk of irritation.

\section{Tetracyclines}

Antibacterials such as tetracycline and clindamycin are the offending agents in over $50 \%$ of the reported cases of drug-induced esophageal disorders. ${ }^{81}$ Drug-induced esophagitis caused by tetracycline or derivates is often manifested in double-contrast studies by the development of small, discrete ulcers, typically in the mid-esophagus near the level of the aortic arch or left main bronchus. ${ }^{82}$ Crowson et al ${ }^{65}$ treated three patients with esophageal ulceration associated with the ingestion of tetracycline or its derivative doxycycline, and found a strong temporal relationship between taking the capsules and the onset of the esophageal ulceration. They recommended an alternative to this class, when possible, for patients with any esophageal obstructive element, and advised patients not to take them within 1 hour of going to bed.

Gencosmanoglu et $\mathrm{al}^{83}$ described two different clinical patterns of esophageal injury induced by tetracycline or doxycycline, comparing these patterns with respect to demographic, endoscopic, and clinical characteristics of the patients. They analyzed 48 patients diagnosed with doxycycline- or tetracycline-induced esophageal injury who were divided into two groups according to the type and location of their esophageal lesions. They found that mid-esophageal ulceration was induced predominantly by doxycycline, whereas distal esophagitis was induced by tetracycline. This work highlighted that the type of tetracyclines taken by patients may provide physicians with some clues about the pattern of esophageal injury, because midesophageal ulceration seems to be more frequently associated with doxycycline, and distal esophagitis candidiasis with other tetracyclines.

In another study, five cases of doxycycline-induced esophagitis with endoscopic images were reported. ${ }^{84}$ The authors demonstrated a strong correlation between esophagitis and how the medicines were taken. Swallowing the medicine with a small amount of water and recumbent posture after ingestion were the main predisposing factors, especially in the case of potentially harmful medications such as tetracyclines. ${ }^{84}$

\section{Legal and ethical considerations}

In hospital geriatric units, compounding is very common, but recently, serious concerns have been raised about the safety of this practice, with legal and ethical ramifications. Like any medical activity, the administration of solid oral dosage forms to dysphagic patients is burdened by the possible materialization of unwanted events for the patient. Since any manipulation of a medicinal product that is not clearly described in the product instructions is an off-label use of the product, the prescriber becomes legally responsible for any adverse effect that may ensue. ${ }^{34,85}$ The off-label use of 
medicines must be supported by documented scientific evidence and requires the patient's informed consent. ${ }^{86}$

The prescriber has an ethical responsibility to provide patients with the information they need to make free and conscious decisions about their health care. In the case of elderly patients, who generally tend to passively accept physicians' proposals, such communication is an especially important part of the doctor-patient relationship. Doctors must explain in understandable terms the limits of the medical intervention and the possible consequences, describe the advantages and risks that could arise from the therapy, make it clear that manipulation of the dosage form of the medicine is an off-label use, and obtain the patient's informed consent.

Obtaining informed consent may be complicated in geriatric patients, and even more so in dysphagic ones because of the clinical conditions linked to or responsible for the swallowing disorder. Additional issues may come from the coexistence of neurologic disorders or postoperative cognitive dysfunction. ${ }^{17}$

These are the aspects of great ethical and professional interest in working with a segment of the population that is socially, physically, and mentally vulnerable. ${ }^{87}$ The legal ramifications of off-label use, the ethical requirements to explain in understandable terms to patient's and to obtain informed consent from those who may have limited cognitive abilities pose a considerable challenge to prescribers.

\section{Concluding remarks}

The concomitant presence of different acute and chronic diseases in geriatric patients obliges physicians to prescribe a large number of medicines, most of which are sold as solid oral dosage forms; there is an almost complete absence of age-appropriate formulations that would allow personalization of therapy, especially in patients with dysphagia. Thus, HCPs in geriatric units or home care quite often manipulate solid formulations to facilitate their administration, a practice that in most cases is an off-label use of the medicine prescribed. Since the path to having geriatric tailored medicinal products on the market is neither simple nor short, physicians, in the absence of reliable alternatives, are obliged to prescribe solid oral dosage forms and advise their compounding in the hospital pharmacy.

However, before taking this hazardous route, alternative solutions and possible adverse events must be carefully evaluated. The prescriber should consider the possibility of substituting the solid with a liquid dosage form or of using alternative routes of administration in a case-by-case manner. If this is not feasible, the physician, in close collaboration with the pharmacist, should evaluate the risk-to-benefit ratio of administering a compounded marketed formulation. Pharmacists should have a pivotal role in this decision, since they have a solid background in biopharmaceutics, pharmaceutical technology, and drug delivery. In this evaluation, important factors to be taken into consideration are the formulation technology, especially whether the formulation is a modified-release form (which in most cases would exclude its manipulation), and whether the APIs have a narrow therapeutic window or are irritants for the GIT mucosa. If it is decided that the manipulation of a prolonged-release dosage form is truly necessary, the dose should be evaluated carefully to avoid dose dumping. If the compounding of a gastro-resistant tablet or capsule has to be performed, particular attention should be paid to the reason/s for the presence of an enteric coating. Gastric mucosa irritation, drug inactivation in gastric fluids, and inconstant or limited absorption in the stomach are the possible motivations.

If the final decision is to compound a marketed formulation, the prescriber should discuss this decision with the patient, describing the documented scientific evidence on the efficacy and safety of the off-label use of the drug (not very common in the literature), and must obtain informed consent. Then, a licensed physician or pharmacist, and not a nurse or another HCP, must perform compounding, assuring minimal API loss and the correctness of the dose in the compounded form.

Since the problem of oral therapy in geriatric dysphagic patients is not new, reputable institutions have issued guidelines on marketed product compounding. ${ }^{88-91}$ In the absence of published studies on the manipulation of a specific medicinal product, prescribers and pharmacists should refer to the general principles reported in them. Unfortunately, this issue receives minimal attention by the stakeholders and, only recently, regulatory agencies and academics are taking the first steps toward the development of age-appropriate geriatric medicinal products. ${ }^{59,92}$ In May 2011, the European Medical Agency's Committee for Medicinal Products for Human Use established the Geriatric Expert Group to provide scientific advice on different matters related to geriatric medicines and gerontology. Among others, the Group has to provide advice on "specific geriatric aspects of medicine development, assessment of products or pharmacovigilance issues". ${ }^{93}$

We sincerely hope that these actions will improve the quality aspects of medicines for older people and reduce the risks linked to the manipulation of marketed medicinal products.

\section{Acknowledgment}

The authors would like to thank Sheila Beatty for editing the English usage in the manuscript. 


\section{Disclosure}

The authors report no conflicts of interest in this work.

\section{References}

1. Vaupel JW. Biodemography of human ageing. Nature. 2010; 464(7288):536-542.

2. Stegemann S, Ecker F, Maio M, et al. Geriatric drug therapy: neglecting the inevitable majority. Ageing Res Rev. 2010;9(4):384-398.

3. Singh S, Bajorek B. Pharmacotherapy in the ageing patient: the impact of age per se (a review). Ageing Res Rev. 2015;24(Pt B):99-110.

4. Nobili A, Garattini S, Mannucci P. Multiple diseases and polypharmacy in the elderly: challenges for the internist of the third millennium. $J$ Comorbidity. 2011;1(1):28-44.

5. Hajjar ER, Cafiero AC, Hanlon JT. Polypharmacy in elderly patients. Am J Geriatr Pharmacother. 2007;5(4):345-351.

6. Stegemann S, Gosch M, Breitkreutz J. Swallowing dysfunction and dysphagia is an unrecognized challenge for oral drug therapy. Int $J$ Pharm. 2012;430(1-2):197-206.

7. Humbert IA, Robbins J. Dysphagia in the elderly. Phys Med Rehabil Clin N Am. 2008;19(4):853-866, ix-x.

8. Fusco S, Cariati D, Schepisi R, et al. Management of oral drug therapy in elderly patients with dysphagia. J Gerontol Geriatr. 2016;64(1):9-20.

9. Langmore SE, Terpenning MS, Schork A, et al. Predictors of aspiration pneumonia: how important is dysphagia? Dysphagia. 1998;13(2):69-81.

10. Roy N, Stemple J, Merrill RM, Thomas L. Dysphagia in the elderly: preliminary evidence of prevalence, risk factors, and socioemotional effects. Ann Otol Rhinol Laryngol. 2007;116(11):858-865.

11. Kawashima K, Motohashi Y, Fujishima I. Prevalence of dysphagia among community-dwelling elderly individuals as estimated using a questionnaire for dysphagia screening. Dysphagia. 2004;19(4):266-271.

12. Holland G, Jayasekeran V, Pendleton N, Horan M, Jones M, Hamdy S. Prevalence and symptom profiling of oropharyngeal dysphagia in a community dwelling of an elderly population: a self-reporting questionnaire survey. Dis Esophagus. 2011;24(7):476-480.

13. Hughes SM. Management of dysphagia in stroke patients. Nurs Older People. 2011;23(3):21-24.

14. Perry L, Love CP. Screening for dysphagia and aspiration in acute stroke: a systematic review. Dysphagia. 2001;16(1):7-18.

15. Daniels SK. Neurological disorders affecting oral, pharyngeal swallowing. GI Motil. 2006.

16. Perry L. Screening swallowing function of patients with acute stroke. Part one: identification, implementation and initial evaluation of a screening tool for use by nurses. J Clin Nurs. 2001;10(4):463-473.

17. Setacci C, Sirignano A, Ricci G, Spagnolo AG, Pugliese F, Speziale F. A new ethical and medico-legal issue: vascular surgery and the postoperative cognitive dysfunction. J Cardiovasc Surg (Torino). 2015 56(4):607-615.

18. Nilsson H, Ekberg O, Olsson R, Hindfelt B. Quantitative aspects of swallowing in an elderly nondysphagic population. Dysphagia. 1996;11(3):180-184.

19. Rivlin MM. Protecting elderly people: flaws in ageist arguments. $B M J$. 1995;310(6988):1179-1182.

20. Etzioni DA, Liu JH, Maggard MA, Ko CY. The aging population and its impact on the surgery workforce. Ann Surg. 2003;238(2):170-177.

21. Marengoni A, Angleman S, Melis R, et al. Aging with multimorbidity: a systematic review of the literature. Ageing Res Rev. 2011;10(4):430-439.

22. Schiele JT, Quinzler R, Klimm H-D, Pruszydlo MG, Haefeli WE. Difficulties swallowing solid oral dosage forms in a general practice population: prevalence, causes, and relationship to dosage forms. Eur J Clin Pharmacol. 2013;69(4):937-948.

23. Stubbs J, Haw C, Dickens G. Dose form modification - a common but potentially hazardous practice. A literature review and study of medication administration to older psychiatric inpatients. Int Psychogeriatr. 2008;20(3):616-627.

24. Haw C, Stubbs J. Administration of medicines in food and drink: a study of older inpatients with severe mental illness. Int Psychogeriatr. 2010;22(3):409-416.
25. US Food and Drug. Available from: http://www.fda.gov/Drugs/ GuidanceComplianceRegulatoryInformation/PharmacyCompounding/ ucm339764.htm\#what. Accessed December 1, 2015.

26. Bonacucina G, Logrippo S, Cespi M, et al. Chemical and microbiological stability studies of an aqueous solution of pravastatin sodium salt for drug therapy of the dysphagic patients. Eur J Hosp Pharm. 2016; 23(5):288-293.

27. Sestili M, Ferrara L, Logrippo S, Ganzetti R. Detection of medication errors in hospital discharge communications of patients on enteral nutrition. Nutr Ther Metab. 2014;32(3):152-154.

28. Kyle G. Medication management in older people with dementia. J Community Nurs . 2012;26:31.

29. Alldred D, Standage C. Medication errors in care homes. Nurs Times. 2010;107(24):14-15.

30. Bennett B, Howard C, Barnes H, Jones A. Medication management in patients with dysphagia: a service evaluation. Nurs Stand. 2013;27(41): $41-48$.

31. Kelly J, Wright D. Administering medication to adult patients with dysphagia: part 2. Nurs Stand. 2010;24(26):61-68.

32. Kelly J, Wright D, Wood J. Medicine administration errors in patients with dysphagia in secondary care: a multi-centre observational study. J Adv Nurs. 2011;67(12):2615-2627.

33. Verrue C, Mehuys E, Boussery K, Remon JP, Petrovic M. Tablet-splitting: a common yet not so innocent practice. $J$ Adv Nurs. 2011;67(1): $26-32$

34. James A. The legal and clinical implications of crushing tablet medication. Nurs Times. 2003;100(50):28-29.

35. Kelly J, Eggleton A, Wright D. An analysis of two incidents of medicine administration to a patient with dysphagia. J Clin Nurs. 2011; 20(1-2):146-155.

36. Cornish P. "Avoid the crush": hazards of medication administration in patients with dysphagia or a feeding tube. CMAJ. 2005;172(7):871-872.

37. Beckwith MC, Feddema SS, Barton RG, Graves C. A guide to drug therapy in patients with enteral feeding tubes: dosage form selection and administration methods. Hosp Pharm. 2004;39(3):225-237.

38. Boada M, Arranz FJ. Transdermal is better than oral: observational research of the satisfaction of caregivers of patients with alzheimer's disease treated with rivastigmine. Dement Geriatr Cogn Disord. 2013; 35(1-2):23-33.

39. Salmon D, Pont E, Chevallard H, et al. Pharmaceutical and safety considerations of tablet crushing in patients undergoing enteral intubation. Int J Pharm. 2013;443(1-2):146-153.

40. Sestili M, Logrippo S, Cespi M, et al. Inappropriate prescribing of solid oral dosage forms in elderly dysphagic patients: a retrospective study. Eur J Clin Pharmacol. 2017.

41. Florence AT. An Introduction to Clinical Pharmaceutics. 1st ed. Press P, editor. London: Pharmaceutical Press; 2010.

42. Buckton G, Beezer AE. The relationship between particle size and solubility. Int J Pharm. 1992;82(3):R7-R10.

43. Ridolfo AS, Thompkins L, Bechtol LD, Carmichael RH. Benoxaprofen, a new anti-inflammatory agent: particle-size effect on dissolution rate and oral absorption in humans. J Pharm Sci. 1979;68(7):850-852.

44. Zafar MU, Farkouh ME, Fuster V, Chesebro JH. Crushed clopidogrel administered via nasogastric tube has faster and greater absorption than oral whole tablets. J Interv Cardiol. 2009;22(4):385-389.

45. Henney HR, Fitzpatrick A, Stewart J, Runyan JD. Relative bioavailability of tizanidine hydrochloride capsule formulation compared with capsule contents administered in applesauce: a single-dose, open-label, randomized, two-way, crossover study in fasted healthy adult subjects. Clin Ther. 2008;30(12):2263-2271.

46. Manrique YJ, Lee DJ, Islam F, et al. Crushed tablets: does the administration of food vehicles and thickened fluids to aid medication swallowing alter drug release? J Pharm Pharm Sci. 2014;17(2):207-219.

47. Royal Society of Chemistry. Implications of altering oral solid-dose formulations; 2011. Available from: http://dysphagia-medicine.com/. Accessed April 7, 2016.

48. Oral dosage forms that should not be crushed 2015. Available from: www.ismp.org/tools/donotcrush.pdf. Accessed November 12, 2015. 
49. Do Not Rush to Crush/Society of Hospital Pharmacists Australia. Available from: http://www.shpa.org.au/Publications/Do-not-Rush-to-Crush. Accessed April 7, 2016.

50. Schier JG, Howland MA, Hoffman RS, Nelson LS. Fatality from administration of labetalol and crushed extended-release nifedipine. Ann Pharmacother. 2003;37(10):1420-1423.

51. Ferron GM, Ku S, Abell M, et al. Oral bioavailability of pantoprazole suspended in sodium bicarbonate solution. Am J Health Syst Pharm. 2003;60(13):1324-1329.

52. Dentinger PJ, Swenson CF, Anaizi NH. Stability of pantoprazole in an extemporaneously compounded oral liquid. Am J Health Syst Pharm. 2002;59(10):953-956.

53. Committee for Medicinal Products for Human use (CHMP). Guideline on the pharmacokinetic and clinical evaluation of modified release dosage forms (EMA/CPMP/EWP/280/96 Corr1). 2013:6. Available from: http://www.ema.europa.eu/docs/en_GB/document_library/Scientific_ guideline/2013/03/WC500140482.pdf. Accessed December 10, 2015.

54. EMA/CHMP/EWP/280/96, (CHMP) C for MP for HU. Guideline on the Pharmacokinetic and Clinical Evaluation of Modified Release Dosage Forms (EMA/CPMP/EWP/280/96 Corr1). London: 2014.

55. ISMP. To chew, or not to chew? Patient died after chewing medication; 2016. Available from: https://www.ismp.org/consumers/chewable.asp. Accessed April 7, 2016.

56. Gudin J, Levy-Cooperman N, Kopecky EA, Fleming AB. Comparing the effect of tampering on the oral pharmacokinetic profiles of two extended-release oxycodone formulations with abuse-deterrent properties. Pain Med. 2015;16(11):2142-2151.

57. Habib WA, Alanizi AS, Abdelhamid MM, Alanizi FK. Accuracy of tablet splitting: comparison study between hand splitting and tablet cutter. Saudi Pharm J. 2014;22(5):454-459.

58. Messina R, Becker R, van Riet-Nales DA, Stegemann S. Results from a preliminary review of scientific evidence for appropriateness of preparations, dosage forms and other product design elements for older adult patients. Int J Pharm. 2015;478(2):822-828.

59. De Spiegeleer B, Wynendaele E, Bracke N, et al. Regulatory development of geriatric medicines: to GIP or not to GIP? Ageing Res Rev. 2016;27:23-36.

60. Jain V, Pitchumoni CS. Gastrointestinal side effects of prescription medications in the older adult. J Clin Gastroenterol. 2009;43(2):103-110.

61. Liabeuf S, Gras V, Moragny J, Laroche ML, Andrejak M. Ulceration of the oral mucosa following direct contact with ferrous sulfate in elderly patients: a case report and a review of the French National Pharmacovigilance Database. Clin Interv Aging. 2014;9:737-740.

62. Graham DY. What the gastroenterologist should know about the gastrointestinal safety profiles of bisphosphonates. Dig Dis Sci. 2002;47(8):1665-1678.

63. Bonavina L, DeMeester TR, McChesney L, Schwizer W, Albertucci M, Bailey RT. Drug-induced esophageal strictures. Ann Surg. 1987;206(2): 173-183.

64. Fennerty MB. NSAID-related gastrointestinal injury. Evidence-based approach to a preventable complication. Postgrad Med. 2001;110(3): 87-88, 91-94.

65. Crowson TD, Head LH, Ferrante WA. Esophageal ulcers associated with tetracycline therapy. JAMA. 1976;235(25):2747-2748.

66. Abraham SC, Yardley JH, Wu TT. Erosive injury to the upper gastrointestinal tract in patients receiving iron medication: an underrecognized entity. Am J Surg Pathol. 1999;23(10):1241-1247.

67. Cimino-Mathews A, Broman JH, Westra WH, Illei PB. Iron pill-induced tumefactive mucosal injury of the hypopharynx. Am J Surg Pathol. 2010;34(11):1720-1722.

68. Areia M, Gradiz R, Souto P, et al. Iron-induced esophageal ulceration. Endoscopy. 2007;39(Suppl 1):E326.

69. Kim ST, Kaisar OM, Clarke BE, et al. "Iron lung": distinctive bronchoscopic features of acute iron tablet aspiration. Respirology. 2003;8(4): 541-543.

70. Jinbu Y, Demitsu T. Oral ulcerations due to drug medications. Jpn Dent Sci Rev. 2014;50(2):40-46.
71. Rosenthal T, Adar R, Militianu J, Deutsch V. Esophageal ulceration and oral potassium chloride ingestion. Chest. 1974;65(4):463-465.

72. Zografos GN, Georgiadou D, Thomas D, Kaltsas G, Digalakis M. Drug-induced esophagitis. Dis Esophagus. 2009;22(8):633-637.

73. Sinar DR, Bozymski EM, Blackshear JL. Effects of oral potassium supplements on upper gastrointestinal mucosa: multicenter clinical comparison of three formulations and placebo. Clin Ther. 1986;8(2):157-163.

74. Pusztaszeri MP, Genta RM, Cryer BL. Drug-induced injury in the gastrointestinal tract: clinical and pathologic considerations. Nat Clin Pract Gastroenterol Hepatol. 2007;4(8):442-453.

75. Singh G. Recent considerations in nonsteroidal anti-inflammatory drug gastropathy. Am J Med. 1998;105(1B):31S-38S.

76. Cryer B. Nonsteroidal anti-inflammatory drug gastrointestinal toxicity. Curr Opin Gastroenterol. 2001;17(6):503-551.

77. Somasundaram S, Simpson R, Rafi S, Shergill JK, Bjarnason I, Wrigglesworth J. 2,4-Diamino-6-hydroxy pyrimidine inhibits NSAIDs induced nitrosyl-complex EPR signals and ulcer in rat jejunum. $B M C$ Gastroenterol. 2002;2:1-7.

78. Bjarnason I, Zanelli G, Prouse P, et al. Blood and protein loss via smallintestinal inflammation induced by non-steroidal anti-inflammatory drugs. Lancet. 1987;2(8561):711-714.

79. Gibson GR, Whitacre EB, Ricotti CA. Colitis induced by nonsteroidal anti-inflammatory drugs. Report of four cases and review of the literature. Arch Intern Med. 1992;152(3):625-632.

80. Bott S, Prakash C, McCallum RW. Medication-induced esophageal injury: survey of the literature. Am J Gastroenterol. 1987;82(8):758-763.

81. Jaspersen D. Drug-induced oesophageal disorders: pathogenesis, incidence, prevention and management. Drug Saf. 2000;22(3):237-249.

82. Levine MS. Drug-induced, caustic, and radiation esophagitis. In: Radiology of the Esophagus. Texas: Society of Abdominal Radiology; 1989:73-89.

83. Gencosmanoglu R, Kurtkaya-Yapicier O, Tiftikci A, Avsar E, Tozun N, Oran ES. Mid-esophageal ulceration and candidiasis-associated distal esophagitis as two distinct clinical patterns of tetracycline or doxycycline-induced esophageal injury. J Clin Gastroenterol. 2004;38(6):484-489.

84. Kadayifci A, Gulsen MT, Koruk M, Savas MC. Doxycycline-induced pill esophagitis. Dis Esophagus. 2004;17(2):168-171.

85. Griffith R, Griffiths H, Jordan S. Administration of medicines. Part 1: the law and nursing. Nurs Stand. 2003;18(2):47-53; quiz 54, 56.

86. Minghetti P, Palmieri I, Selmin F. When authorized medicinal products are not available: possible alternatives to meet legitimate expectations of patients. J Pharm Heal Serv Res. 2010;1(3):107-112.

87. Mueller PS, Hook CC, Fleming KC. Ethical issues in geriatrics: a guide for clinicians. Mayo Clin Proc. 2004;79(4):554-562.

88. Nissen L. Australian Don't Rush to Crush Handbook. 1st ed. The Society of Hospital Pharmacists of Australia. Australia: Collingwood Victoria; 2013.

89. Mitchell JF. Oral dosage forms that should not be crushed. ISMP. 2015:1-15. Available from: http://www.ismp.org/tools/donotcrush.pdf. Accessed April 7, 2016.

90. Wright D, Chapman N, Foundling-Miah M, et al. Consensus Guideline on the Medication Management of Adults with Swallowing Difficulties Supplement Produced in Association with Consensus Guideline on the Medication Management of Adults with Swallowing Difficulties. Chesham Bucks, UK: MGP Ltd Location; 2006.

91. Wrexham Maelor Hospital. General guidelines for administration of medications to patients with swallowing difficulties. Available from: http://www.newtguidelines.com/SwallowingDifficulties.html. . Chesham Bucks, UK: MGP Ltd Location.

92. EMA/165974/2013, (CHMP) C for MP for HU. Concept Paper on the Need for a Reflection Paper on Quality Aspects of Medicines for Older People. London, UK: 2013.

93. EMA/281009/2013, Evaluation HMD and. Mandate, Objectives and Rules of Procedure for the CHMP Geriatric Expert Group (GEG). London, UK: 2013. 
Clinical Interventions in Aging

\section{Publish your work in this journal}

Clinical Interventions in Aging is an international, peer-reviewed journal focusing on evidence-based reports on the value or lack thereof of treatments intended to prevent or delay the onset of maladaptive correlates of aging in human beings. This journal is indexed on PubMed Central, MedLine,

CAS, Scopus and the Elsevier Bibliographic databases. The manuscript management system is completely online and includes a very quick and fair peer-review system, which is all easy to use. Visit http://www.dovepress. $\mathrm{com} /$ testimonials.php to read real quotes from published authors.

Submit your manuscript here: http://www.dovepress.com/clinical-interventions-in-aging-journal 